\title{
Analysis of Equalizer Performance against Bit Error Rate in Filter Bank Multicarrier System with AWGN and Multipath Channel
}

\author{
Mahdif Indiarto \\ Gunadarma University \\ Jakarta, Indonesia
}

\author{
Hartono Siswono \\ Gunadarma University \\ Jakarta, Indonesia
}

\begin{abstract}
Filter-Bank Multicarrier (FBMC) is a signal processing technique using filter-bank in the data transmission process. FBMC-OQAM system has a better BER value than FBMCQAM but has interference. This can result in a decrease in the quality of the transmission process which results in reduced signal quality. So, it is necessary to handle this problem, one of which is the addition of an equalizer to the FBMC system. Signal quality can be seen by calculating the Bit Error Rate (BER) and Signal to Noise Ratio (SNR) values. System simulation was performed using MATLAB R2017a software. The system consists of a transmitter as a modulator, a receiver as a demodulator, and a channel. The FBMC system without an equalizer on the AWGN channel produces a lower BER value at the same SNR value compared to other system schemes with $\mathrm{BER}=0$ at $\mathrm{SNR} 19 \mathrm{~dB}$. The equalizer shows good performance in FBMC systems with the Multipath ETU channel. Where there is an improvement in the BER value at SNR $\geq 7 \mathrm{~dB}$. In the AWGN channel, the addition of an equalizer to the FBMC system results in a higher BER value than the FBMC system without an equalizer. So that the addition of an equalizer on the FBMC system with the AWGN channel is not recommended.
\end{abstract}

\section{Keywords}

FBMC, Bit Error Rate, Equalizer, AWGN, Multipath.

\section{INTRODUCTION}

In the digital era, telecommunications is not only limited to cellular communications. But it also covers other fields, one of which is IoT (Internet of Things) and Big Data. So that in the future telecommunication technology, a system with higher data speed and capacity is needed as well as a more efficient allocation of spectrum usage than the current system. In addition, a system that has good signal quality and efficiency is also needed.

There are several candidate waveform schemes for future telecommunications technology, namely Orthogonal Frequency Division Multiplexing (OFDM), Universal Filtered Multi-Carrier (UFMC), Generalized Frequency Division Multiplexing (GFDM), and Filter Bank Multicarrier (FBMC). FBMC is a signal processing technique by utilizing a filter bank in the data transmission process. The FBMC system has advantages in terms of the efficiency of the allocation of spectral use [1]. In FBMC, there are several bank filter application schemes on the FBMC system, namely FS-FBMC and PPN-FFT FBMC. Based on previous research, the use of the polyphase network (PPN) filters on bank filters can reduce the level of computational complexity in the FBMC system [2].

Offset Quadrature Amplitude Modulation (OQAM) is a modulation technique that is widely used in FBMC systems. By using OQAM modulation, the FBMC system has lower inter-carrier interference (ICI) effect than OFDM, UFMC, and GFDM [3]. In addition, the FBMC-OQAM system has greater flexibility for use in the $5 \mathrm{G}$ wave scenario. FBMC-OQAM produces lower BER than other systems [4]. The FBMC system with Pulse Amplitude Modulation (PAM) modulation has advantages in terms of compatibility because it has the same symbol rate as the OFDM system [5]. The FBMC system with Quadrature Amplitude Modulation (QAM) modulation has a better spectral efficiency than the CPOFDM system. In addition, the FBMC-QAM system does not have interference when compared to the FBMC-OQAM system [6]. On the other hand, the FBMC-OQAM system has a lower Bit Error Rate (BER) value compared to the FBMCQAM system [7]. So that the FBMC-OQAM system has better transmission quality than the FBMC-QAM system.

As is well known in the FBMC-OQAM system, besides interference from noise during the transmission process, there is also interference in the form of interference. This can result in a decrease in the quality of the transmission process which results in reduced signal quality. So, it is necessary to handle this problem, one of which is the addition of an equalizer to the FBMC system. This paper will discuss the effect of the Equalizer on the performance of the FBMC system as measured by the values of Bit Error Rate (BER), and Signal to Noise Ratio (SNR).

\section{METHODOLOGY}

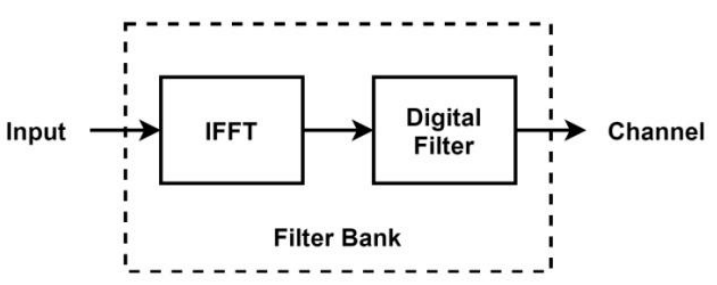

Figure 1. Basic Filter Bank System 


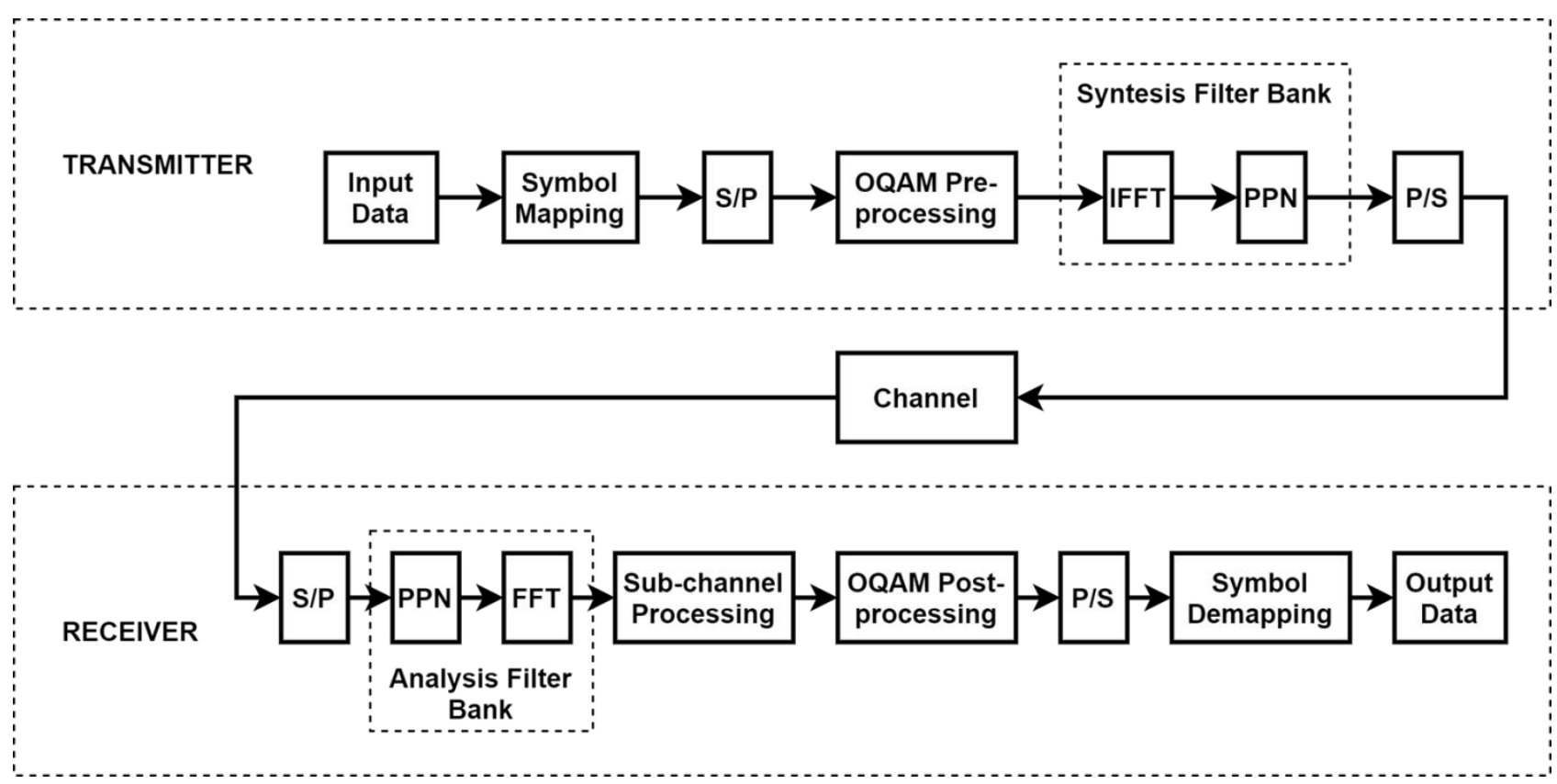

Figure 2. PPN-FFT FBMC Transceiver

Filter Bank Multicarrier or also known as FBMC is the first multicarrier modulation scheme based on a Filter-Bank. FBMC was developed in 1960 by Chang. FBMC was created to fix the shortcomings experienced in Orthogonal Frequency Division Multiplexing or OFDM, one of which is the use of a cyclic prefix [8].

The basic Filter-Bank system consists of IFFT/FFT and digital filters as shown in IFFT/FFT functions as a multicarrier modulator on the input and a multicarrier demodulator on the output. The use of multicarrier modulation techniques provides an advantage in the use of spectrum because it is divided into smaller frequencies [9].

\subsection{PPN-FFT FBMC Transmitter}

The transmitter functions as a modulator which modulates the signal generated by the random data generator into a modulated signal. The PPN-FFT FBMC transmitter system consists of several parts, namely symbol mapping, serial to parallel conversion, OQAM Pre-Processing, Synthesis Filter Bank (consists of IFFT and PPN), and parallel to serial conversion as shown in Figure 2.

Input data is data that will be processed and sent by the transmitter as an information signal. The data is generated by a random data generator in the form of binary code arranged randomly with values 0 and 1 . In the symbol mapping section, the signal will be modified where the bits will be converted into symbols according to the M-ary value. Furthermore, in the FBMC system, the data will be converted from serial to parallel. Modulated data is converted into parallel series. Conversion to parallel allows information data to be sent at the same time.

The OQAM process on the PPN-FFT FBMC transmitter is depicted in Figure 3. The QAM offset modulation (OQAM) process is carried out by transmitting the real and imaginary parts of the QAM symbol data with an offset or shift of T/2 samples or half the subcarrier interval. And a phase rotation of pi/2 $\left(90^{\circ}\right)$ is carried out between adjacent sub-carriers and sub-symbols. In this process, a complex to real conversion is carried out, where the real and imaginary parts of the complex valued symbol are separated to form a new symbol. The order of the symbols is based on the sub-channel number, that is, the different conversions between even and odd sub-channels. At $\mathrm{k}$ with an odd value, the real part is given a delay of 1 symbol, while at $\mathrm{k}$ is even, the imaginary part is given a delay of 1 symbol. This process increases the sample rate by 2 times compared to before. After that, multiply by $\theta_{k, n}$ with $\theta_{k, n}=\exp (j \pi / 2(k+n))=j^{(k+n)}$ [10]. In this process, preamble signal insertion is also carried out at the beginning of the symbol array. The preamble signal is used as a guide signal for the parameter or phase initialization at the receiver for the timing recovery process.

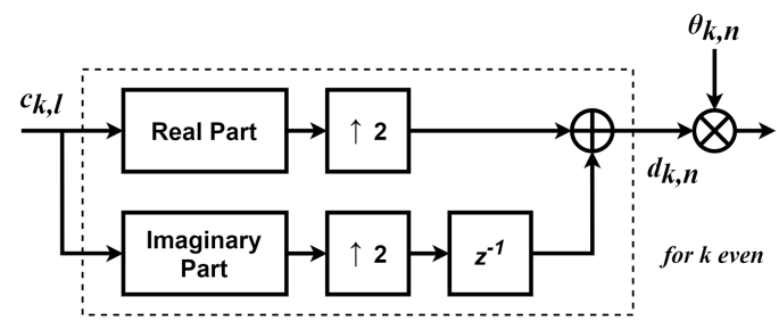

Complex to Real Conversion

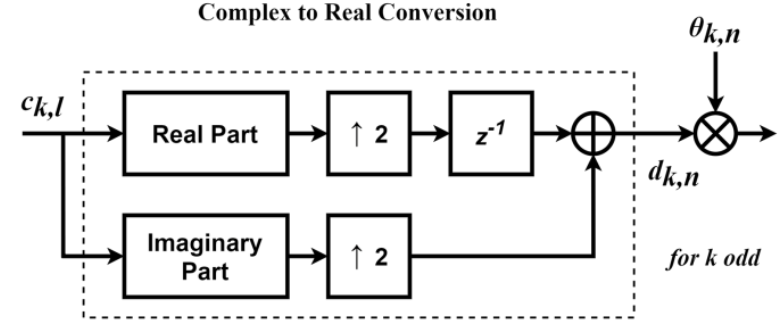

Complex to Real Conversion

Figure 3. OQAM Pre-Processing Block Diagram

Synthesis Filter-Bank (SFB) consists of IFFT and PPN sections. Previously, the data will be multiplied by the $\beta$ multiplier on SFB with the value of $\beta_{k, n}=(-1)^{k n}(-1)^{k K}$ for the filter length KM-1 [10]. The Inverse Fast Fourier Transform (IFFT) process will convert the signal from the frequency domain to the time domain. In this process, a polyphase filter 
is applied to the signal in the time domain. The filter used is the PHYDYAS finite impulse response (FIR) prototype filter with an overlapping factor of $\mathrm{K}=2,3$, or 4 . The data conversion process from parallel to series is formed from the up-sampler and delay processing on the Synthesis Filter Bank. The modulated signal is then transmitted through the channel.

\subsection{PPN-FFT FBMC Receiver}

The receiver functions as a demodulator. The receiver will demodulate the modulated signal received from the channel to convert it into real information data. The PPN-FFT FBMC receiver system block consists of several parts as shown in Figure 2.

The received data signal is then converted back from series to parallel. The serial to the parallel conversion process in the PPN-FFT FBMC system is formed from the down-sampler and delay processes carried out by the Filter Bank Analysis. Analysis Filter-Bank (AFB) is the reverse process of the filter bank synthesis (SFB). Where the AFB consists of the PPN and FFT sections. The PPN process on the receiver will apply a polyphase filter to the signal just like the PPN process on the transmitter.

The FFT process is the opposite of the IFFT process at the transmitter. FFT will convert data from the time domain to the frequency domain. After the FFT process is carried out, the data is then multiplied by the $\beta$ multiplier on AFB with the value of $\beta_{k, n}=(-1)^{k n}(-1)^{k K}=\beta^{*}{ }_{k, n}$ for the filter length KM-1 [10]. In the sub-channel process, a preamble removal process is carried out that was previously inserted in the transmitting process. So that by doing this process the information data signal is obtained without preamble signal. In addition, in this section an Equalization process is also carried out if desired.

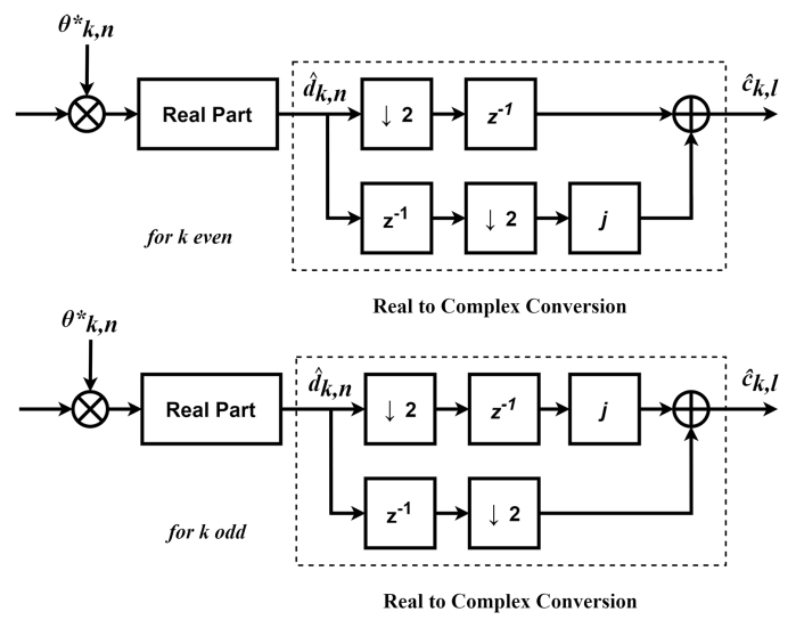

Figure 4. OQAM Post-Processing Block Diagram

OQAM Post-Processing is carried out by converting from real numbers to complex numbers as shown in Figure 4 . The process is carried out based on $\mathrm{k}$ which is odd and even. In the OQAM process, the multiplication process by $\theta_{k, n}^{*}$ is the first time followed by the actual part-taking operation. Where is $\theta_{k, n}^{*}=\exp (-j \pi / 2(k+n))=(-j)^{(k+n)}[10]$. Then the conversion is carried out from real to complex numbers to produce numbers that contain imaginary values. Converting real to complex numbers reduces the sample rate by factor of 2 . After the OQAM process, the data is converted from parallel to series. So that the data to be demodulated is in the form of a series. The resulting data is then recovered to produce the actual bit series with symbol demapping based on M-ary. This process is carried out by separating the carrier signal generated by the mapping process on the transmitter. So that a bit series is produced that corresponds to the information signal sent. The output data is an information data signal that has passed the demodulation process, the resulting data signal is expected to be the same as the input data.

\subsection{AWGN and Multipath Channel}

In communication theory, it is assumed that the transmitted signal is distorted by noise. The most common noise to assume is additive Gaussian noise or also called additive white Gaussian noise (AWGN) channel. Additive means added. The Gaussian is following the Gaussian distribution or also called the normal distribution. Meanwhile, this noise is called white because it consists of all the frequencies in the spectrum as white light. AWGN modeling is simple so it is very efficient to use in simulations, especially MATLAB [11].

In a wireless mobile communication system, signals can travel from transmitter to receiver via multiple reflective paths; this phenomenon is referred to as multipath propagation. These effects can cause fluctuations in the amplitude, phase, and angle of arrival of the received signal, giving rise to the terminology of multipath fading [12]. The propagation conditions for multipath fading are determined by a combination of the multipath delay profile and the maximum Doppler frequency $\left(f_{D}\right)$ of 5,70 , or $300 \mathrm{~Hz}$. In addition, a Doppler frequency of $200 \mathrm{~Hz}$ was specified for the uplink time adjustment performance requirements. There are several multipath channel models, especially for wireless applications, including EPA, EVA, and ETU. In this paper using the Multipath ETU channel.

The Extended Typical Urban (ETU) channel is used to generate channel models for wireless applications based on the GSM Urban Model. The ETU has a maximum Doppler frequency of $300 \mathrm{~Hz}$. This channel model has a total of nine channels and a maximum delay of 5000ns. This channel model has a limited bandwidth of $20 \mathrm{MHz}$. The UE channel model has a speed of $120 \mathrm{Km} / \mathrm{h}$ or $350 \mathrm{Km} / \mathrm{h}$. This model applies to urban areas in general. The delay profile of the ETU channel model can be seen in Table 1 .

Table 1. ETU Delay Profile [13]

\begin{tabular}{|c|c|}
\hline Excess Tap Delay (ns) & Relative Power dB \\
\hline 0 & -1.0 \\
\hline 30 & -1.0 \\
\hline 120 & -1.0 \\
\hline 200 & 0.0 \\
\hline 230 & 0.0 \\
\hline 500 & 0.0 \\
\hline 1600 & -3.0 \\
\hline 2300 & -5.0 \\
\hline 5000 & -7.0 \\
\hline
\end{tabular}

\section{ANALYSIS AND RESULT}

The simulation was carried out using MATLAB R2017b software with the parameters in Table 2. The simulation resulted in the calculation of the Bit Error Rate (BER) against the Signal to Noise Ratio (SNR) which is displayed in the form of a plot diagram in Figure 5. 
Table 2. Simulation Parameters

\begin{tabular}{|c|l|c|}
\hline No & \multicolumn{1}{|c|}{ Parameter } & Value \\
\hline 1 & Modulation & 16-OQAM \\
\hline 2 & FFT Size & 64 \\
\hline 3 & SNR & $0-20 \mathrm{~dB}$ \\
\hline 4 & Channel & $\begin{array}{c}\text { AWGN and } \\
\text { Multipath ETU }\end{array}$ \\
\hline 5 & Overlapping Factor (K) & 4 \\
\hline 6 & Equalizer & 3-tap \\
\hline
\end{tabular}

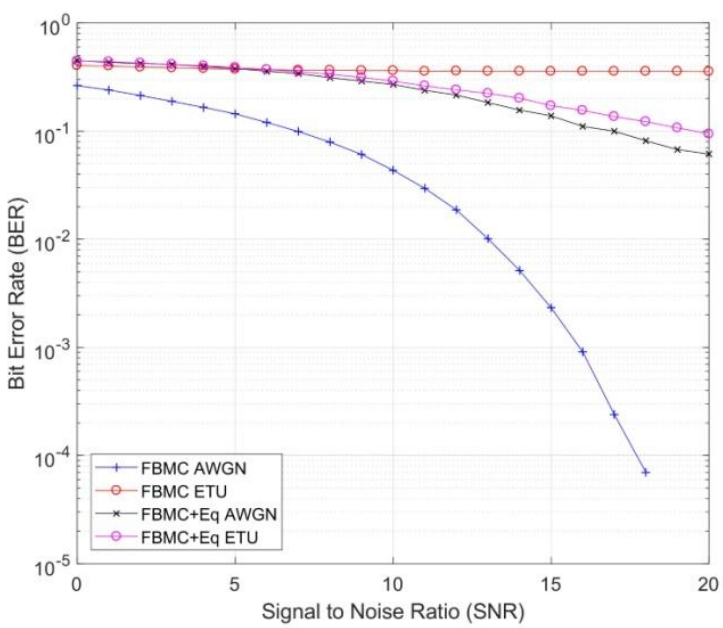

Figure 5. BER and SNR Plot Diagram

Based on the plot diagram generated from the simulation, it is known that the FBMC system on the AWGN channel produces a lower BER value than other systems at the same SNR value. In addition, from the plot diagram, it can also be seen that as the SNR value increases, the BER value decreases, except for the FBMC system on the Multipath ETU channel, there is no visible decrease in the BER value. So that to make it easier to carry out the analysis, the diagram data generated from the simulation are converted in tabular form which can be seen in Table 3 .

Table 3. Comparison of BER and SNR Value

\begin{tabular}{|c|c|c|c|c|}
\hline \multirow{2}{*}{$\begin{array}{c}\text { SNR } \\
(\mathbf{d B})\end{array}$} & \multicolumn{4}{|c|}{ BER } \\
\cline { 2 - 5 } & $\begin{array}{c}\text { FBMC } \\
\text { AWGN }\end{array}$ & $\begin{array}{c}\text { FBMC } \\
\text { ETU }\end{array}$ & $\begin{array}{c}\text { FBMC+EQ } \\
\text { AWGN }\end{array}$ & $\begin{array}{c}\text { FBMC+EQ } \\
\text { ETU }\end{array}$ \\
\hline 0 & 0.26357 & 0.40516 & 0.44841 & 0.44498 \\
\hline 1 & 0.24014 & 0.40055 & 0.43291 & 0.43933 \\
\hline 2 & 0.21368 & 0.39084 & 0.42223 & 0.42741 \\
\hline 3 & 0.18851 & 0.38544 & 0.41275 & 0.41463 \\
\hline 4 & 0.16591 & 0.37940 & 0.39606 & 0.40123 \\
\hline 5 & 0.14454 & 0.37502 & 0.37827 & 0.38821 \\
\hline 6 & 0.12030 & 0.37102 & 0.35823 & 0.37127 \\
\hline 7 & 0.09922 & 0.36704 & 0.34108 & 0.35429 \\
\hline & & & & \\
\hline
\end{tabular}

\begin{tabular}{|c|c|c|c|c|}
\hline 8 & 0.07924 & 0.36517 & 0.31216 & 0.33565 \\
\hline 9 & 0.06091 & 0.36315 & 0.28952 & 0.31379 \\
\hline 10 & 0.04345 & 0.36218 & 0.26954 & 0.29027 \\
\hline 11 & 0.02963 & 0.35788 & 0.23846 & 0.26210 \\
\hline 12 & 0.01873 & 0.35941 & 0.21523 & 0.24083 \\
\hline 13 & 0.01013 & 0.35852 & 0.18354 & 0.22310 \\
\hline 14 & 0.00516 & 0.35904 & 0.15618 & 0.20153 \\
\hline 15 & 0.00233 & 0.35695 & 0.13838 & 0.17173 \\
\hline 16 & 0.00091 & 0.35777 & 0.11052 & 0.15587 \\
\hline 17 & 0.00024 & 0.35700 & 0.09999 & 0.13659 \\
\hline 18 & 0.00007 & 0.35752 & 0.08169 & 0.12255 \\
\hline 19 & 0.00000 & 0.35770 & 0.06753 & 0.10735 \\
\hline 20 & 0.00000 & 0.35652 & 0.06154 & 0.09402 \\
\hline
\end{tabular}

Table 3 shows that the FBMC system on the AWGN channel produces the lowest BER value compared to other systems. On the AWGN channel, the lowest BER value is BER $=0$ at SNR 19dB, in other words, the transmission is carried out without any bit errors. The FBMC system on the Multipath ETU channel produces the lowest BER value of 0.35652 at SNR 20dB. In addition, in the FBMC system on the Multipath ETU channel, there was an increase in the BER value at SNR $12 \mathrm{~dB}$ and $14 \mathrm{~dB}$. The FBMC system with the addition of an equalizer on the AWGN channel produces the lowest BER value of 0.06154 at SNR 20dB. The FBMC system with the addition of an equalizer on the Multipath ETU channel produces the lowest BER value of 0.09402 at SNR 20dB.

Overall, from all these systems, it is known that the BER value decreases along with the increasing SNR value. Where the best BER value $\mathrm{BER}=0$ at $\mathrm{SNR} 19 \mathrm{~dB}$ is obtained by the FBMC system on the AWGN channel. The FBMC system on the Multipath channel shows a decrease in the BER value as the SNR value increases, except for the $12 \mathrm{~dB}$ and $14 \mathrm{~dB}$ SNR value where there is an increase in the BER value. However, this decline is not too significant, so it is not seen in Figure 5.

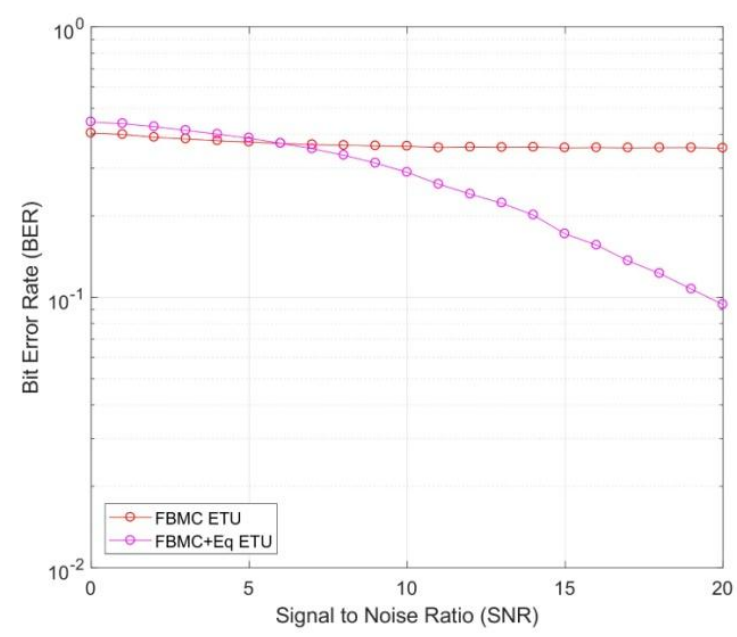

Figure 6. Comparison of BER and SNR values on Multipath ETU channel 
Based on the simulation results, an analysis of the use of Equalizer was also carried out on each channel. Figure 6 shows that the addition of an equalizer to the FBMC system with the Multipath ETU channel has an effect on the BER value. Where in the Multipath ETU channel the BER value generated by the system with the addition of the Equalizer is lower than without Equalizer at SNR values of $7 \mathrm{~dB}$ and above. However, at SNR values below $7 \mathrm{~dB}$, the BER value obtained by the system without Equalizer is better than the system with Equalizer.

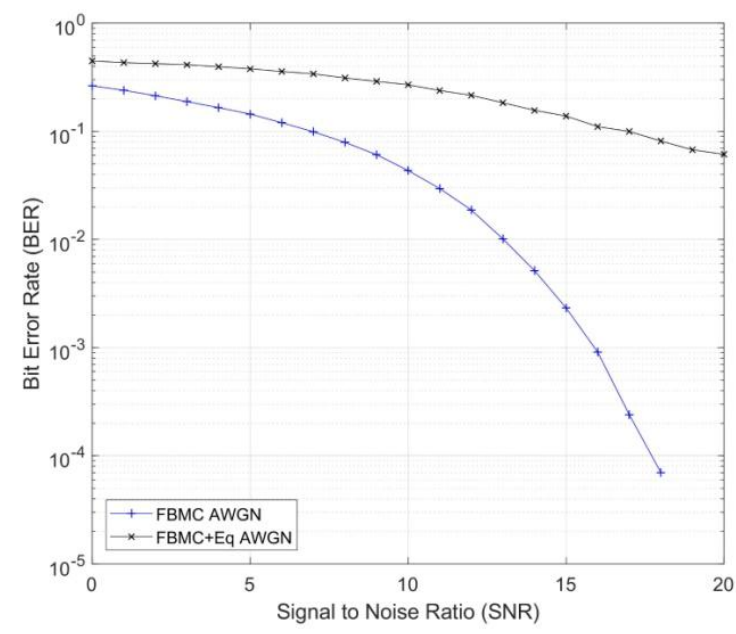

Figure 7. Comparison of BER and SNR values on AWGN channel

As for the FBMC system with AWGN channels, the use of the Equalizer is not recommended. The comparison of BER and SNR values on the AWGN channel can be seen in Figure 7. The simulation results show that the use of the Equalizer on the AWGN channel results in an increase in the BER value at the same SNR value so that the Equalizer is not effectively used by the FBMC system on the AWGN channel.

\section{CONCLUSION}

Based on the discussion and analysis of the test results data that have been carried out, it can be seen that the FBMC system without an equalizer on the AWGN channel produces lower BER values at the same SNR value compared to other system schemes. The equalizer shows good performance in FBMC systems with the Multipath ETU channel. Where there is a decrease in the BER value at $\mathrm{SNR} \geq 7 \mathrm{~dB}$. As for the AWGN channel, the addition of an equalizer to the FBMC system increases the BER value compared to the FBMC system without an equalizer. So that the addition of an equalizer on the FBMC system with the AWGN channel is not recommended.

In future research, it is hoped that other channel models can be used to see the further effect of the equalizer on the quality of signal transmission. In addition, the use of equalizer types or other techniques can be considered to get better results.

\section{ACKNOWLEDGMENTS}

The author would like to thank Gunadarma University, my family, and all those who have support in completing this paper, both morally and materially. Especially my advisor, Mr. Hartono Siswono, who has provided guidance and advice to the author so that this final project can be completed properly.

\section{REFERENCES}

[1] Bellanger, M., et al., "FBMC physical layer : a primer", ICT-PHYDYAS Project, 2010, 1-31.

[2] Gerzaguet, R., et al., "The 5G candidate waveform race: a comparison of complexity and performance", EURASIP Journal an Wireless Communications and Networking, 2017:13.

[3] Khrouf, W., Siala M., Abdelkefi F., "How Much FBMC/OQAM Is Better than FBMC/QAM? A Tentative Response Using the POPS Paradigm", Wireless Communications and Mobile Computing, Vol 2018, 2018.

[4] Doré, Jean-Baptiste, et.al., Waveform contenders for 5G: Description, analysis and comparison. Physical Communication Elsevier, Vol. 24, 2017, 46-61.

[5] Mattera, D., et.al. "Filter Bank Multicarrier with PAM Modulation for Future Wireless Systems". Elsevier: Signal Processing, 2015.

[6] Nam, H., et.al. "A New Filter-Bank Multicarrier System with Two Prototype Filters for QAM Symbols Transmission and Reception". IEEE Transaction on Wireless Communication, 2016.

[7] Kim, J., et.al. "A New Filter-Bank Multicarrier System: The Lineary Processed FBMC System". IEEE Transaction on Wireless Communication, 2018.

[8] Kaur, Satwinder, et al., "Survey of Filter Bank Multicarrier (FBMC) as an efficient waveform for 5G", International Jurnal of Pure and Applied Mathematics. Vol. 118, 2018, 45-49.

[9] Stevan Hendrawan Lodo, "Gabungan Modulasi OQAM Dan Pulse Shaping Pada Sistem OFDM", Universitas Mercu Buana, Jakarta, 2007

[10] Viholainen, A., Bellanger, M., Huchard, M., "D5.1 Prototype filter and structure optimization", ICTPHYDYAS, 2009.

[11] Lund University, "Chapter 10 Gaussian Channel", Faculty of Engineering (LTH) Lund University, Lund.

[12] Sklar, B., "Rayleigh Fading Channels in Mobile Digital Communication Systems Part I: Characterization", IEEE Communications Magazine, 1997, 90-100.

[13] ETSI, "LTE; Evolved Universal Terrestrial Radio Access (E-UTRA); Base Station (BS) radio transmission and reception (3GPP TS 36.104 version 14.3.0 Release 14)", European Telecommunications Standards Institute, 2017 